\title{
Diagnóstico nutricional de crianças de zero a cinco anos atendidas pela rede municipal de saúde em área urbana da região sul do Brasil, 1988*
}

\section{Nutritional diagnosis of children under five years of age assisted by the municipal health network an urban area in the Southern Region of Brazil, 1988}

\author{
Erica Ell**, Lydia M.M. Loureiro**, Silvia A. Gugelmin**, Sônia M.R. Cruz**
}

\begin{abstract}
ELL, E. et al. Diagnóstico nutricional de crianças de zero a cinco anos atendidas pela rede municipal de saúde em área urbana da regiāo sul do Brasil, 1988. Rev. Saúde públ., S.Paulo, 26: 217-22, 1992. Como um dos requisitos para a operacionalização do Sistema de Vigilância Alimentar e Nutricional (SISVAN) no município de Curitiba, PR, Brasil, foi realizado um levantamento epidemiológico retrospectivo com dados de 1988 , que buscou através do exame antropométrico conhecer a prevalência e as formas de desnutrição calórico-protéico de crianças menores de cinco anos atendidas pela Rede Municipal de Saúde de Curitiba. Numa amostra de 4.213 crianças encontrou-se pela Classificação de Gòmez $28,1 \%$ de desnutridos, sendo que 3,6\% destes situam-se no grau II e III. A Classificação de Seoane e Latham modificada por Batista Filho revelou que $19,7 \%$ das crianças apresentam alguma forma de desnutrição. O perfil antropométrico revelou maior concentração de crianças no primeiro decil a partir do sexto mês de vida, sendo esta mais acentuada no grupo etário de 12 a 24 meses para os índices: peso/idade e altura/idade.
\end{abstract}

Descritores: Desnutrição protéico-calórica, epidemiologia. Antropometria. Estado nutricional.

\section{Introdução}

Entre as principais carências nutricionais do país, com amplas conseqüências para a saúde, encontra-se a desnutrição energético-protéica, a qual está relacionada com a ingestão e utilização biológica de alimentos, doenças infecto-parasitárias, bem como com a estrutura socioeconômica e política do pais. Desta forma, ela retrata as diferenças sociais e econômicas dos indivíduos no interior de uma sociedade ${ }^{9,10,14}$.

A tomada de medidas antropométricas é um dos métodos mais utilizados no acompanhamento do crescimento e desenvolvimento de crianças, não só em função da praticidade, baixo custo e precisão, mas também pela sua importância no diagnóstico precoce da desnutrição, pois a primeira manifestação deste déficit é a perda ponderal ${ }^{4,10,11}$.

* Financiado pelo Projeto CADS/ARAUCÁRIA-UFPR Fundação kellogg's - Convênio 98/87 e pela Secretaria Municipal de Saúde de Curitiba - Paraná; apresentado no "Seminánio de Vigilância Alimentar e Nutricional ${ }^{*}$, em Curitiba, PR

** Secretaria Municipal de Saúde - Curitiba, PR - Brasil e Universidade Federal do Paraná - Curitiba, PR - Brasil

Separatas/Reprints: S.A. Gugelmin - Ladeira Ary Barroso, 19

- Ap. 302 - 22010-060 - Rio de Janeiro, RJ - Brasil
$\mathrm{Na}$ rotina diária das unidades de sáude normalmente são realizados registros de medidas antropométricas, fornecendo ao serviço informações contínuas sobre o estado nutricional da população atendida. Estes dados por si só, ou quando combinados com outros indicadores de setores ligados à economia, educação e agricultura, podem subsidiar melhor compreensão da problemática nutricional e efetivar políticas de intervenção.

Diante da precária situação de saúde das populações de países em desenvolvimento, tem-se discutido métodos para promover melhoras destes quadros. Dentre as várias propostas, o Sistema de Vigilância Alimentar e Nutricional (SISVAN), tem-se consolidado como instrumento para o conhecimento da situação alimentar e nutricional de uma população, subsidiando a formulação de políticas, programas e intervenções, contribuindo assim para a construção da seguridade alimentar (Mason ${ }^{6}, 1984$ ).

Segundo a Organização Mundial da Saú$\mathrm{de}^{12}$ (1976) a "Vigilância alimentar e nutricional deve proporcionar informações contínuas sobre a situação nutricional da população, bem como, os fatores que a influenciam. Essas informações servirão de base para formu- 
lar políticas, efetuar a planificação e a administração dos programas de melhora de consumo alimentar e do estado nutricional".

Dentro da finalidade do SISVAN o presente estudo tem como propósito demonstrar e determinar o estado nutricional de crianças atendidas pelos centros de saúde da rede municipal de Curitiba, assim como auxiliar na implantação deste sistema no município buscando os determinantes desta situação e formulando, com a participação da comunidade, políticas de intervenção em nível individual e coletivo.

\section{Metodologia}

O objeto de estudo deste trabalho foram os prontuários de 4.213 crianças menores de cinco anos que freqüentavam os 41 centros de saúde da Secretaria Municipal de Saúde de Curitiba, em funcionamento durante 1988, de onde se retiraram os seguintes dados: identificação (número da ficha familiar), sexo, data de nascimento, data de visita, peso e altura. Esses dados foram coletados pelas equipes de área, treinadas pelas chefias locais e com supervisão de profissionais do nível central, que utilizaram o critério aleatório sistemático seguindo intervalo amostral definido pelo número da ficha familiar e amostra do centro de saúde.

Para se chegar a esta amostragem empregou-se o método estratificado com partilha proporcional, e seu tamanho foi definido através da fórmula de variância para distribuição binomial, assumindo um erro de $5 \%$ e intervalo de confiança de $95 \%$ para cada centro de saúde, acrescentando $10 \%$ na amostra para cobrir erros de coleta.

Foi efetuado um teste piloto que demonstrou algumas dificuldades. Sendo assim, optou-se por redesenhar a amostra, o que reduziu a aproximadamente $30 \%$ o número inicial de cada centro de saúde, obtendo-se como total 4.598 prontuários.

A elaboração da metodologia de coleta, o teste piloto e a coleta propriamente dita foram realizados por uma equipe multidisciplinar da Secretaria Municipal de Saúde com assessoria de profissionais de outras instituições. A compilação e análise dos dados coletados foram realizadas pelas autoras do presente estudo.

Do total de 41 centros de saúde, dois não enviaram os dados, o que restringiu o número de centros de saúde analisados a 39 . Devido a não compilação desses dados, a exclusão de dados com erros de coleta, valores extremos, e outros, a amostra final ficou em 4.213 crianças, o que representou uma perda de $8,4 \%$ da amostra total.

A compilação dos dados foi realizada através do "Anthropometric Software Package $^{\prime \prime}$, que utiliza o padrão de referência do $\mathrm{Na}$ tional Center for Health Statistic (NCHS), recomendado pela $\mathrm{OMS}^{3}$.

A avaliação do estado nutricional foi realizada a partir dos índices antropométricos de peso/idade, altura/idade e peso/altura. As crianças foram classificadas em seis faixas etárias, sendo que até os dois anos de idade foram divididas de seis em seis meses, pois o crescimento nesta faixa se dá de forma mais acelerada; após, foram agrupadas de ano em ano.

$O$ perfil antropométrico foi empregado para demonstrar a situação global do estado nutricional da população infantil. Ele é estruturado a partir da distribuição das crianças conforme os índices peso/idade e altura/idade em intervalos decilares que se relacionam com o padrão de referência. Em cada decil há 10\% dos indivíduos da população-referência. Sendo assim, a população estudada deve acompanhar esta linha de normalidade. Em situação de déficit nutricional haverá maior concentração de crianças nos primeiros decis, ocasionando no gráfico uma inclinação das colunas para a esquerda. Em casos de sobrepeso, a inclinação das colunas dar-se-á para a direita. Assim, tanto no déficit quanto no sobrepeso haverá uma freqüência superior aos $10 \%$ esperados da população-referência, nos respectivos decis ${ }^{8,9}$.

Para estimar a prevalência da desnutrição optou-se pela Classificação de Gòmez que indica, de acordo com o valor do peso mediano esperado para a idade e sexo, formas leves (grau I), moderadas (grau II) e graves (grau III) de desnutrição, quando esse valor estiver situado entre 76 a $90 \%, 60$ a $75 \%$ e abaixo de $60 \%$ da mediana, respectivamente ${ }^{7}$.

Para especificar as formas de desnutrição em relação a sua duração, utilizou-se a Classificação de Seoane e Latham modificada por Batista Filho, que utiliza os índices altura/idade e peso/altura, expressos em termos de desvios percentuais da mediana, considerando como desnutrição recente quando a altura/idade estiver $>92 \%$ e o peso/altura $<90 \%$ do valor mediano esperado, desnutrição pregressa quando a altura/idade estiver $<92 \%$ e o peso/idade $>90 \%$ e desnutrição 
Tabela 1. Distribuição percentual de crianças menores de 05 anos atendidas pela Rede Municipal de Saúde de Curitiba segundo faixa etária e estado nutricional (Classificação de Gómez) - 1988.

\begin{tabular}{|c|c|c|c|c|c|c|}
\hline \multirow{3}{*}{$\begin{array}{c}\text { Faixa } \\
\text { Etária } \\
\text { (meses) }\end{array}$} & \multirow{2}{*}{\multicolumn{2}{|c|}{ Nutridas }} & \multirow{2}{*}{\multicolumn{2}{|c|}{ Desnutridas }} & \multirow{2}{*}{\multicolumn{2}{|c|}{ Total }} \\
\hline & & & & & & \\
\hline & $n$ & $\%$ & $n$ & $\%$ & $n$ & $\%$ \\
\hline $00 \cdot 06$ & 887 & 74,4 & 305 & 25,6 & 1.192 & 100 \\
\hline $06 \cdot 12$ & 408 & 69,7 & 176 & 30,3 & 586 & 100 \\
\hline $12 \cdot 18$ & 287 & 65,8 & 149 & 34,2 & 436 & 100 \\
\hline $18 \cdot 24$ & 280 & 70,2 & 119 & 29,8 & 399 & 100 \\
\hline $24 \cdot 36$ & 575 & 75,7 & 185 & 24,3 & 760 & 100 \\
\hline $36 \cdot 60$ & 593 & 70,6 & 247 & 29,4 & 840 & 100 \\
\hline Total & 3.030 & 71,9 & 1.183 & 28,1 & 4.213 & 100 \\
\hline$=0,05$ & \multicolumn{4}{|c|}{$x^{2} g l_{5}=11,07$} & \multicolumn{2}{|c|}{$x^{2} \mathrm{cal}=19,80$} \\
\hline
\end{tabular}

crônica quando a altura/idade estiver $<92 \%$ e o peso/altura $<90 \%$ do valor mediano esperado da população referência ${ }^{l}$.

Para o tratamento estatístico dos resultados encontrados nas classificações, utilizou-se $o$ teste do qui-quadrado ${ }^{2,15}$.

\section{Resultados}

O perfil antropométrico mostra que até o sexto mês de vida as crianças estão situadas próximas à normalidade, havendo um pequeno déficit no índice altura/idade mostrando que algumas crianças estão com a altura abaixo do normal para sua idade, o que pode estar relacionado a fatores genéticos ou fatores adversos durante o período gestacional e período pós-natal.

A partir do sexto mês de vida percebe-se que tem início um déficit estatural e ponderal progressivo. As crianças com situação nutricional mais comprometida estão nas faixas de 12 a 18 e 18 a 24 meses, sendo o déficit ponderal maior na primeira, enquanto que a segunda apresenta um déficit estatural.
A distribuição do peso e da altura nas faixas etárias seguintes mantém uma inclinação à esquerda nos índices altura/idade, porém inferior ao da faixa de 12 a 24 meses.

$\mathrm{Na}$ Tabela 1 as crianças estão distribuídas segundo a idade e o estado nutricional (Classificação de Gòmez). Observa-se que $28,1 \%$ das crianças estudadas apresentam algum déficit ponderal. A aplicação do teste quiquadrado demonstrou uma associação estatisticamente significativa entre a idade e o estado nutricional, com um nível de significância de $5 \%$.

O grau I de desnutrição é predominante em relação aos demais (Tabela 2). Relacionando as crianças desnutridas situadas nos graus II e III com o total da amostra (4.213 crianças) encontra-se 3,6\%.

A Tabela 3 apresenta os resultados obtidos pela Classificação de Seoane e Latham modificada por Batista Filho. Verifica-se que 19,7\% das crianças apresentam alguma forma de desnutrição. Utilizando-se essa classificação, o teste qui-quadrado demostrou que não há associação entre estado nutricional e faixa etária.

Tabela 2. Distribuição percentual de crianças desnutridas menores de 05 anos atendidas pela Rede Municipal de Curitiba segundo faixa etária e graus de desnutrição (Classificação de Gòmez) - 1988.

\begin{tabular}{|c|c|c|c|c|c|c|c|c|}
\hline \multirow{3}{*}{$\begin{array}{c}\text { Faixa } \\
\text { Etária } \\
\text { (meses) }\end{array}$} & \multirow{2}{*}{\multicolumn{2}{|c|}{ Grau I }} & \multirow{2}{*}{\multicolumn{2}{|c|}{ Grau II }} & \multirow{2}{*}{\multicolumn{2}{|c|}{ Grau III }} & \multirow{2}{*}{\multicolumn{2}{|c|}{ Total }} \\
\hline & & & & & & & & \\
\hline & $n$ & $\%$ & $n$ & $\%$ & $n$ & $\%$ & $n$ & $\%$ \\
\hline $00 \cdot 06$ & 256 & 83,9 & 44 & 14,4 & 05 & 1,6 & 305 & 100 \\
\hline $06 \cdot 12$ & 146 & 82,0 & 30 & 16,8 & 02 & 1,1 & 178 & 100 \\
\hline $12 \cdot 18$ & 133 & 89,3 & 16 & 10,7 & $\cdot$ & - & 149 & 100 \\
\hline $18 \cdot 24$ & 108 & 90,8 & 11 & 09,2 & - & - & 119 & 100 \\
\hline $24 \cdot 36$ & 168 & 90,8 & 15 & 08,1 & 02 & 1,1 & 185 & 100 \\
\hline $36 \cdot 60$ & 222 & 89,9 & 25 & 10,1 & - & $\dot{.}$ & 247 & 100 \\
\hline Total & 1.033 & 87,3 & 141 & 11,9 & 09 & 0,8 & 1.183 & 100 \\
\hline$\varepsilon=0,05$ & & $x^{2} g$ & & & & & $x^{2} c$ & 94 \\
\hline
\end{tabular}


Tabela 3 - Distribuição percentual de crianças menores de 05 anos atendidas pela Rede Municipal de Saúde de Curitiba segundo fajxa etária e estado nutricional (Classificação de Seoane-Latham modificado por Batista Filho) - 1988.

\begin{tabular}{|c|c|c|c|c|c|c|}
\hline \multirow{3}{*}{$\begin{array}{c}\text { Faixa } \\
\text { Etária } \\
\text { (meses) }\end{array}$} & \multirow{2}{*}{\multicolumn{2}{|c|}{ Nutridas }} & \multirow{2}{*}{\multicolumn{2}{|c|}{ Desnutrida }} & \multirow{2}{*}{\multicolumn{2}{|c|}{ Total }} \\
\hline & & & & & & \\
\hline & $n$ & $\%$ & $n$ & $\%$ & $n$ & $\%$ \\
\hline $00 \cdot 06$ & 964 & 80,9 & 228 & 19,1 & 1.192 & 100 \\
\hline $06 \cdot 12$ & 467 & 79,7 & 119 & 20,3 & 586 & 100 \\
\hline $12 \cdot 18$ & 334 & 76,6 & 102 & 23,4 & 436 & 100 \\
\hline $18 \cdot 24$ & 325 & 81,4 & 74 & 18,6 & 399 & 100 \\
\hline $24 \cdot 36$ & 626 & 82,4 & 134 & 17,6 & 760 & 100 \\
\hline $36 \cdot 60$ & 668 & 79,5 & 172 & 20,5 & 840 & 100 \\
\hline Total & 3.384 & 80,3 & 829 & 19,7 & 4.213 & 100 \\
\hline
\end{tabular}

Tabela 4 - Distribuição percentual de crianças desnutridas menores de 05 anos atendidas pela Rede Municipal de Saúde de Curitiba segundo faixa etária e tipo de desnutrição (Classificação de Seoane-Latham modificada por Batista Filho) - 1988.

\begin{tabular}{|c|c|c|c|c|c|c|c|c|}
\hline \multirow{3}{*}{$\begin{array}{c}\text { Faixa } \\
\text { Etária } \\
\text { (meses) }\end{array}$} & \multirow{2}{*}{\multicolumn{2}{|c|}{ Recente }} & \multirow{2}{*}{\multicolumn{2}{|c|}{ Progressa }} & \multirow{2}{*}{\multicolumn{2}{|c|}{ Crônica }} & \multirow{2}{*}{\multicolumn{2}{|c|}{ Total }} \\
\hline & & & & & & & & \\
\hline & $n$ & $\%$ & $n$ & $\%$ & $n$ & $\%$ & $n$ & $\%$ \\
\hline 00.06 & 173 & 75,9 & 45 & 19,7 & 10 & 4,4 & 228 & 100 \\
\hline $06 \cdot 12$ & 83 & 69,7 & 31 & 26,0 & 05 & 4,2 & 119 & 100 \\
\hline $12 \cdot 18$ & 74 & 72,5 & 20 & 19,6 & 08 & 7,8 & 102 & 100 \\
\hline $18 \cdot 24$ & 43 & 58,1 & 24 & 32,4 & 07 & 9,5 & 74 & 100 \\
\hline $24 \cdot 36$ & 89 & 66,4 & 34 & 25,4 & 11 & 8,2 & 134 & 100 \\
\hline $36 \cdot 60$ & 87 & 50,6 & 73 & 42,4 & 12 & 7.0 & 172 & 100 \\
\hline Total & 549 & 66,6 & 227 & 27,4 & 53 & 6.4 & 829 & 100 \\
\hline$\Sigma=0,05$ & \multicolumn{4}{|c|}{$\left.x^{2} g\right|_{10}=18,30$} & & & \multicolumn{2}{|c|}{$x^{2}$ cal $=38,00$} \\
\hline
\end{tabular}

A desnutrição recente, na qual a criança apresenta altura/idade normal, porém o peso deficiente, predomina sobre as demais com $66,2 \%$ (Tabela 4). A ocorrência da forma crônica, que significa um comprometimento tanto do peso como da altura, praticamente duplicou a partir do primeiro ano de vida. $\mathrm{Na}$ desnutrição pregressa encontram-se $27,4 \%$ das crianças. Isso mostra um déficit da altura em relação a idade, sugerindo que estas estiveram expostas a condições desfavoráveis no passado, levando a uma desaceleração do crescimento. Pode-se verificar também uma tendência de aumento da desnutrição pregressa com o decorrer da idade. Segundo o teste estatístico, observa-se associação entre a faixa etária e os tipos de desnutrição (Tabela 4).

\section{Discussão}

Ao analisar os dados de crianças menores de cinco anos atendidas nos centros de saúde da Secretaria Municipal de Saúde de Curitiba, constatou-se um déficit nutricional considerá- vel. Segundo a Classificação de Gòmez, $28,1 \%$ das crianças estão desnutridas, sendo que os casos de desnutrição se concentram no grau I $(87,3 \%)$. Entretanto não se pode desconsiderar os graus II e III pois, mesmo em menor proporção, são os graus que representam maior risco de morte para as crianças. A soma desses dois graus quando, relacionada à amostra total (4.213), resultou em $3,6 \%$ sugerindo um problema social importante ${ }^{14}$.

Estes resultados mostram que, em termos comparativos, a população estudada encontrase em situação mais favorável em relação às populações do Terceiro Mundo, as quais apresentam uma prevalência de desnutrição de $50 \%$ ou mais ${ }^{5}$.

A Classificação de Seoane e Latham, modificada por Batista Filho, possui maior especificidade quando comparada com a Classificação de Gòmez, mostrando-se mais adequada para estudos que queiram especificar as formas e a duração da desnutrição. Os resultados obtidos através desta classificação mostram que $19,7 \%$ das crianças estavam desnutridas, sendo a desnutrição recente a mais comum 
$(66,2 \%)$. Isto indica que prevalece o déficit ponderal.

De acordo com o perfil antropométrico, observa-se que a condição nutricional das crianças situadas na faixa de 0 a 6 meses de idade apresenta-se próxima do esperado. No entanto, os valores de peso/idade e altura/idade no primeiro decil aumentam consideravelmente a partir do sexto mês. Possivelmente, na maioria das vezes estas situações advêm de vários fatores, como: um aporte calórico-protéico inadequado, diarréias, infecçōes respiratórias, desmame precoce, introdução inadequada de alimentos e outros condicionantes derivados do quadro de pobreza. Essas crianças necessitam de uma atenção especial para desenvolver todo seu potencial de crescimento. Em casos de intervenção, deve-se em primeira instância verificar as crianças que estão com déficit pondero-estatural, não deixando de dar atenção aos outros grupos, principalmente os que apresentam maior vulnerabilidade biológica, como as crianças menores de um ano?

Este diagnóstico teve como intuito identificar e quantificar a desnutrição na rede municipal de saúde de Curitiba, sendo uma das etapas da implantação e operacionalização do SISVAN. Mesmo sendo a desnutrição um reflexo da interação do indivíduo com o meio em que vive, é importante realizar o levantamento, análise e divulgação periódica dos dados qualitativos, paralelamente aos quantitativos, para que se possa conhecer os determinantes sociais desta situação. A ausência de dados qualitativos referentes a população estudada foi um fator limitante no presente estudo.

Havendo uma rotina de coleta de dados nos diversos setores envolvidos, pode-se adequar o SISVAN aos mesmos, não necessitando criar estruturas próprias para manter o fluxo de informaçōes ${ }^{13}$. Esses dados quando analisados permitirão conhecer a situação alimentar e nutricional e direcionarão as ações para a prevenção e a solução dos possíveis problemas.

\section{Agradecimentos}

- Ao Dr. Malaquias Batista Filho, por sua assessoria no desenvolvimento da pesquisa;

- A Cristina Maria Cantanheda Amarante (Prof'. visitante do DEMQS - ENSP/FIOCRUZ), pela revisão dos cálculos estatísticos.
- À Lana Magaly Pires (Coordenadora do Subprojeto de Nutrição do Projeto CADS Araucária e professora auxiliar do Depart. de Nutrição - UFPR), pelo incentivo à realização do trabalho.

ELL, E. et al. [Nutritional diagnosis of children under five years of age assisted by the municipal health network an urban area in the southern Region of Brazil, 1988]. Rev. Saúde públ., S.Paulo, 26: $217-22,1992$. An epidemiological survey was undertaken to fulfil one of the conditions required for the establishment of the Nutritional

Surveillance System, on the basis of data from 1988. In this survey an anthropometric examination was employed for the purpose of discovering the prevalence and the kinds of protein energy malnutrition among children under five years of age who had been assisted at Health Centers of the Municipal Health Department of Curitiba, Paraná (Brazil). Of a sample population of 4,213 children it was found (by the Gomez criterion) that $28.1 \%$ had malnutrition $3.6 \%$ of this percentage being of second or third degree. By the use of the Seoane-Latham criterion as modified by Batista Filho, $19.7 \%$ of the children presented some kind of malnutrition. The distribution of the sample in centiles showed that it contained an excess of children in the first centiles after 6 th months of age as compared with the reference population. This excess was more evident in the 12 to 24-months age group.

Keywords: Protein-calorie malnutrition,epidemiology. Anthropometry. Nutritional status.

\section{Referências Bibliográficas}

1. BATISTA FILHO, M. et al. Estado nutricional de pré-escolares de comunidade rurais do nordeste brasileiro. Rev.bras.Med., 42: 236-4 1, 1985.

2. HOEL, P.G. Estatística elementar. São Paulo, Ed. Atlas, 1981.

3. JORDAN, M.D. Antropometric software package: tutorial guide and handbook. $2^{\text {nd }}$ ed. Atlanta, Ga., Center for Disease Control, 1987.

4. JORDAN, J.R El lactente de 0 a 2 anos: antropometria y crecimiento. In: Cusminsky, M., ed. et al. Crecimiento y desarrolo: hechos y tendencias. Washington, D.C., Organizacion Panamericana de la Salud, 1988. p. 184-209. (OPAS - Publicacion Cientifica, 510).

5. MARTINS, J.A.R \& CHAIN, R Desnutrição: uma proposta de trabalho para unidades básicas de saúde. Arq. bras. Med., 59: 463-6, 1985.

6. MASON, J.B. et al. Vigilância nutricional. Genebra, Organização Mundial da Saúde, 1984.

7. MINISTÉRIO DA PREVIDÊNCIA E ASSISTÉNCIA SOCIAL (MPAS). Manual para avaliação antropométrica do estado nutricional de crianças até 7 anos de idade. Brasilia, 1983.

8. MONTEIRO, C.A. Estudo das condições de saúde das crianças do Município de São Paulo, SP (Brasil), 1984-1985. II - Antropometria nutricional. Rev. Saúde públ., S.Paulo, 20: 446-53, 1986. 
9. MONTEIRO, C.A. \& BENICIO, M.H.D'A. Estado nutricional e renda. S.Paulo em Perspect., 1(3): $67-70,1987$.

10. MONTEIRO, C.A. Critérios antropométricos no diagnóstico da desnutrição em programas de assistência a criança. Rev. Saúde públ., S.Paulo, 18: $56-63,1984$.

11. MONTEIRO, C.A. Recentes mudanças propostas na avaliação antropométrica do estado nutricional infantil: uma avaliação crítica. Rev.Saúde públ, S.Paulo, 18: 56-63, 1984.

12. ORGANIZACION MUNDIAL DE LA SALUD. Metodologia de la vigilância nutricional. Ginebra, 1976. (OMS - Série de Informes Técnicos, 593).
13. ORGANIZACION DE LAS NACIONES UNIDAS PARA LA AGRICULTURA Y LA ALIMENTACION. Oficina Regional para America Latina y el Caribe. Division de Politica Alimentaria y Nutricional. Manual practico para la instalacion de un sistema de vigilancia alimentaria y nutricional. Santiago del Chile, 1989.

14. SPINELLI, M.A.S. et al. Indicadores das condições nutricionais da região do Polonoroeste. VI - Estudo antropométrico, 1985 (1). Rev. Inst. Med.trop. S. Paulo, 30(5): 197-201, 1988.

15. SWINSCOW, T.D.V. Estatística um ao quadrado. São Paulo, Ed. Manoele Ltda, 1988.

Recebido para publicaçāo em 12/12/1990 Reapresentado em 30/3/1992 Aprovado para publicação em 22/5/1992 